\title{
Tissue-specific transcriptome assemblies of the marine medaka Oryzias melastigma and comparative analysis with the freshwater medaka Oryzias latipes
}

Keng Po Lai ${ }^{1,6^{*+}}$, Jing-Woei Li ${ }^{2+}$, Simon Yuan Wang ${ }^{1,6}$, Jill Man-Ying Chiu ${ }^{5,6}$, Anna Tse ${ }^{1,6}$, Karen Lau ${ }^{1,6}$, Si Lok , Doris Wai-Ting Au ${ }^{4,6}$, William Ka-Fai Tse ${ }^{5}$, Chris Kong-Chu Wong ${ }^{5,6}$, Ting-Fung Chan²,

Richard Yuen-Chong Kong ${ }^{4,6^{*}}$ and Rudolf Shiu-Sun Wu $\mathrm{W}^{1,6^{*}}$

\begin{abstract}
Background: The marine medaka Oryzias melastigma has been demonstrated as a novel model for marine ecotoxicological studies. However, the lack of genome and transcriptome reference has largely restricted the use of O. melastigma in the assessment of in vivo molecular responses to environmental stresses and the analysis of biological toxicity in the marine environment. Although O. melastigma is believed to be phylogenetically closely related to Oryzias latipes, the divergence between these two species is still largely unknown. Using Illumina high-throughput RNA sequencing followed by de novo assembly and comprehensive gene annotation, we provided transcriptomic resources for the brain, liver, ovary and testis of O. melastigma. We also investigated the possible extent of divergence between O. melastigma and O. latipes at the transcriptome level.
\end{abstract}

Results: More than 14,000 transcripts across brain, liver, ovary and testis in marine medaka were annotated, of which 5880 transcripts were orthologous between O. melastigma and O. latipes. Tissue-enriched genes were identified in O. melastigma, and Gene Ontology analysis demonstrated the functional specificity of the annotated genes in respective tissue. Lastly, the identification of marine medaka-enriched transcripts suggested the necessity of generating transcriptome dataset of $O$. melastigma.

Conclusions: Orthologous transcripts between O. melastigma and O. latipes, tissue-enriched genes and O. melastigma-enriched transcripts were identified. Genome-wide expression studies of marine medaka require an assembled transcriptome, and this sequencing effort has generated a valuable resource of coding DNA for a non-model species. This transcriptome resource will aid future studies assessing in vivo molecular responses to environmental stresses and those analyzing biological toxicity in the marine environment.

Keywords: Medaka, Marine and freshwater, Transcriptome assembly, De novo assembly, High-throughput RNA sequencing

\footnotetext{
*Correspondence: balllai@hku.hk; bhrkong@cityu.edu.hk; rudolfwu@hku.hk

${ }^{\dagger}$ Equal contributors

'School of Biological Sciences, Kadoorie Biological Sciences Building, The University of Hong Kong, Pokfulam Road, Hong Kong, SAR, China

${ }^{4}$ Department of Biology and Chemistry, City University of Hong Kong, Tat Chee Avenue, Kowloon, Hong Kong, SAR, China

${ }^{6}$ The State Key Laboratory in Marine Pollution, Hong Kong, China Full list of author information is available at the end of the article
} 


\section{Background}

There is a trend of using small marine fish as models to study the biological impact of environmental pollutants and stresses on marine organisms, which is an important area of ecotoxicological studies [1]. Freshwater fish models, such as zebrafish (Danio rerio) and rainbow trout (Oncorhynchus mykiss), have been widely used for ecotoxicological studies in the freshwater environment. However, their responses to environmental toxins can be completely different in marine fish [2-4]. For example, it has been reported that freshwater species were more sensitive to ammonia and metal compounds whereas marine species were more sensitive to pesticide and narcotic compounds [4]. Such differences indicate that ecotoxicological results from freshwater environments cannot be directly applied to the marine environment [1]. The marine medaka Oryzias melastigma (O. melastigma) is an emerging marine fish model used in the investigation of the response of organisms to pollutants, toxins and stresses in marine environments [5,6]. In fact, $O$. melastigma is already used in a variety of estuarine and marine ecotoxicological studies [7-10], demonstrating their potential in studying the effect of organic chemicals, inorganic chemicals, microorganism and environmental stresses in relation to cardiac toxicity [11], hepatotoxicity [9], neurotoxcity [12], immunotoxicity [10], and so forth. In addition, $O$. melastigma has been adopted by the International Life Sciences Institute (ILSI) Health and Environmental Science Institute (HESI) for embryo toxicity testing. Unfortunately, the use of $O$. melastigma as a model in the assessment of in vivo molecular responses to environmental stresses and for analyzing biological toxicity in the marine environment is largely restricted by the lack of molecular resources for O. melastigma [13].

O. melastigma was previously believed to be phylogenetically closely related to the Japanese freshwater ricefish medaka Oryzias latipes (O. latipes) [1,14], of which a draft genome has been reported [15]. However, even within inbred strains within the O. latipes species group, the genome-wide SNP rate between the Hd-rR and HNI strains is among the highest (3.42\%) of all vertebrate species [15]. Recently, $O$. melastigma and O. latipes were shown to belong to two distinct species groups of medaka [16], suggesting they could be even more divergent. Therefore, there may be a pressing need of a genetic database specifically devoted for the marine medaka O. melastigma.

Here, using Illumina high-throughput RNA sequencing (RNA-Seq) followed by de novo assembly and comprehensive annotation and comparison of the transcriptome dataset, we provide transcriptomic resources, including the brain, liver and gonadal tissues (ovary and testis) of female and male O. melastigma. Our primary goal was to produce a reference set of mRNA sequences for O. melastigma that would facilitate the understanding of the local adaptation, genome evolution and population genetics of medaka.
Additionally, the identification of a set of genes along with their functional annotation in multiple organs of O. melastigma would facilitate the use of marine medaka for ecotoxicology studies. Furthermore, we compared the gene sets of $O$. melastigma and $O$. latipes to determine their possible divergence at the transcriptomic level.

\section{Methods}

Tissue specific transcriptome from of O. melastigma were assembled from high-throughput strand-specific RNASeq. The possible divergence between marine and freshwater medaka at the transcriptome level was assessed by comparisons of sequences deposited in public databases and the assemblies generated in this study. A single consensus transcriptome was generated for gene annotation and inter-organ comparative analysis and marine-tofreshwater medaka transcriptome comparison. The overall workflow of the study is shown in Figure 1.

\section{Medaka maintenance and RNA isolation}

All animal research procedures were approved by the Committee on the Use of Live Animals in Teaching and Research (CULATR) at The University of Hong Kong. The freshwater medaka fish $O$. latipes was gifted by David Hinton's laboratory at Duke University. Marine medaka (O. melastigma) were reared and maintained under optimal growth and breeding conditions, as described in Kong et al. $\left(5.8 \mathrm{mg} \mathrm{O}_{2} \mathrm{~L}^{-1}, 28 \pm 2^{\circ} \mathrm{C}\right.$, pH 7.2 in a 14-h light: 10 -h dark cycle) [1]. The stock of marine medaka used in our experiment was obtained from Interocean Industries (Taiwan) and has been reared in our laboratory for over 10 generations. 1:1 ratio of sexually mature 4-month old male and female medaka were placed in a same tank for external fertilization to take place naturally and the fertilized eggs were collected [17]. At 120 days post fertilization, brain, liver, ovary and testis tissues were dissected from randomly selected male $(n=10)$ and female $(n=10)$ fish. To minimize the variation among individual fish, tissue samples from 10 fish were pooled. Total RNA from pooled tissue samples was extracted using the mirVana $^{\mathrm{TM}}$ isolation kit (Applied Biosystems) and then treated with DNase (Ambion) to remove contaminating genomic DNA. The RNA quality was assessed using the Agilent 2100 Bioanalyzer system, and samples with an RNA Integrity Number (RIN) greater than 9 were used for RNA library construction.

\section{Strand-specific library preparation and transcriptome sequencing}

Sequencing was performed at the Centre for Genomic Sciences, The University of Hong Kong. Total RNA was treated with the RiboMinus Eukaryote Kit for RNA-Seq (Invitrogen, Carlsbad, CA) to remove ribosomal RNA, 


\section{Oryzias melastigma transcriptome assembly workflow}

\section{A) Tissue specific sequencing}

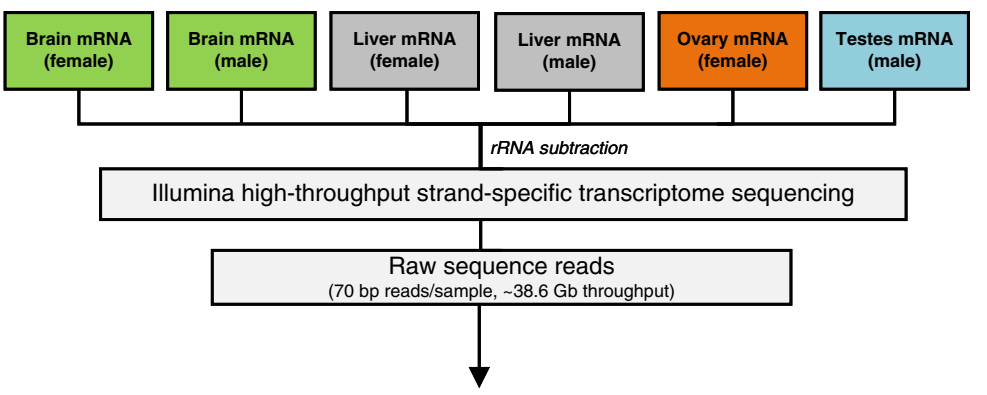

C) Transcriptome assembly and Quality Evaluation

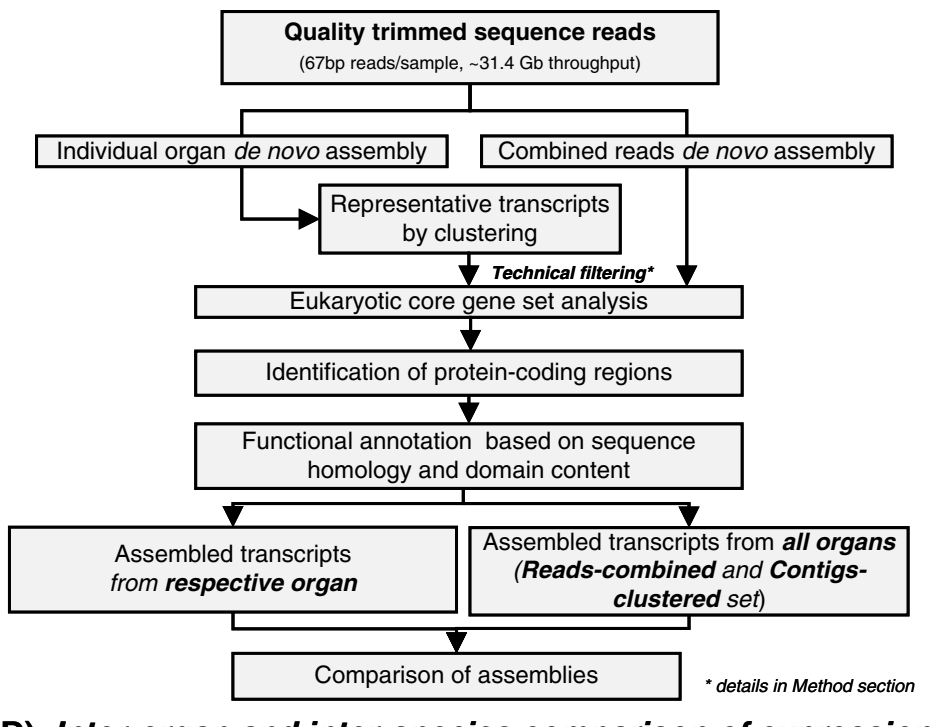

D) Inter-organ and inter-species comparison of expression

\section{B) Divergence between marine and freshwater Medaka}

Comparison of sequences deposited in public database and assemblies generated in this study
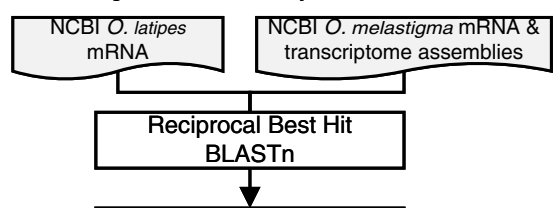

Nucletotide identity

between orthologues

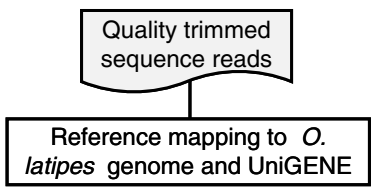

\section{Comparison of assemblies}

with Oryzias latipes and melastigma sequences via Reciprocal BLAST

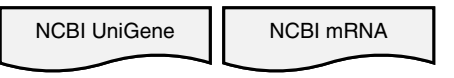

Annotation of assemblies

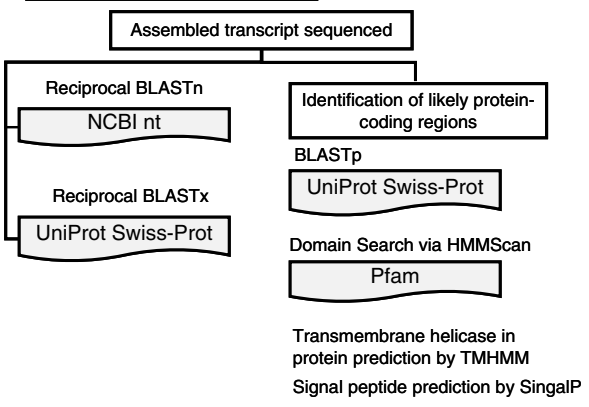

Signal peptide prediction by SingalP
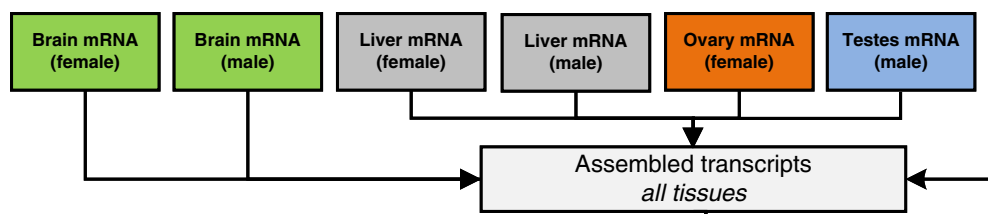

Organ enriched expression of genes

Marine Medaka specific genes relative to Fresh Water Medaka

Figure 1 Workflow of this study. A) Organ-specific transcriptome sequencing using the Illumina GAllx platform. B) Overview of the procedure to investigate the divergence between marine and freshwater medaka at the transcriptome level. C) Pipeline of de novo transcriptome assembly. Refer to main text for details. D) Comparison of inter-organ transcription and marine-to-freshwater medaka.

and the rRNA-depleted RNA was concentrated by ethanol precipitation in the presence of a glycogen carrier (Ambion). The dUTP strand-specific library construction protocol was used to generate templates for Illumina DNA sequencing. Briefly, strand specificity was maintained by the incorporation of deoxy-UTP during second-strand cDNA synthesis and subsequent destruction of the uridine-containing strand during the following step of library construction. The sequencing library was constructed using GAIIx with the use of the Paired-End Cluster Generation Kit v5 and Sequencing Kit v5 (Applied Biosystems) following the manufacturer's recommended protocol, which generated 76-bp-long paired-end sequence reads. The insert size was approximately $200 \mathrm{bp}$. 


\section{Transcriptome assembly}

The sequence reads were dynamically trimmed according to BWA's $-\mathrm{q}$ algorithm with a parameter of 30. A running sum algorithm was executed. Briefly, an cumulative area plot is plotted from 3'-end to the 5'-end sequence reads, where positions of base-calling Phred quality lower than 30 causes an increase of area and vice versa. Such plot was built for each read individually. The read would be trimmed from the 3 '-end to the position where the area was greatest [18]. Read pairs were then synchronized such that all read pairs with sequences of at least $35 \mathrm{bp}$ on both sides after quality trimming were retained and any singleton read resulted from reads trimming were removed. The quality-trimmed sequence reads were assembled using Trinity (r2013-02-25) [19], which uses fixed $k$-mer to generate assembly and is efficient in recovering full-length transcripts and spliced isoforms [19]. Trinity was used rather than multi $k$-mer tools because Trinity was shown to reconstruct the most full-length transcripts for genes expressed in different dynamic ranges when compared with the various single $k$-mer assemblers, while multi $k$-mer tools tended to assemble more artificially fused transcripts [20]. De novo assembly by Trinity was individually performed for each organ and gender. For brain and liver, an additional gender-pooled de novo sequence read assembly was performed. Such gender-pooled assemblies were used to facilitate comparison of tissue enriched genes based on annotation of the assembled transcripts (section Tissue-enriched genes in O. melastigma). Assembled transcripts from individual samples were merged and duplicates were then removed using CD-hit-est [21] (v4.5.4) using the accurate mode (-g 1) with other parameters left as default to yield the final assembly (Contigs-clustered Assembly). CD-Hit uses an incremental clustering algorithm to first sort all assembled transcripts in order of decreasing length. The longest transcript becomes the representative of the first cluster. Then, each remaining transcript is compared to the representatives of all existing clusters and would be clustered to the most similar cluster if the similarity is above threshold of global sequence identity of $\geq 90 \%$. Otherwise a new cluster is defined with that sequence being the representative [22]. Such a merging process broadens the coverage of assemblies produced by Trinity. A de novo meta-tissue assembly (Reads-combined Assembly) [23] was also performed using a virtual library by merging sequence reads from all organs (see also discussion below and Figure 1).

\section{Assembly validation and transcript annotation}

We employed an internal validation approach for mapping quality-trimmed sequence reads back to the assembly to identify poor-quality and potentially misassembled transcripts. Through the process, transcripts with an average base coverage of less than one were removed from the assembly sets. The quality of the assembled transcripts was then assessed using the metric that was suggested for de novo transcriptome assembly [24], including contig count, percentage of reads used in contig, base-pairs in contig, average contig coverage, average contig length and contig N50 length. The quality of the assembly was further assessed by comparison with the 248 core eukaryotic genes (CEGs) [25] with the use of BLASTp, an e-value cut-off of $1.0 \times 10^{-6}$ [26,27] and a requirement of more than $70 \%$ alignment length for the CEGs.

In the first step of transcript annotation, the assembled transcripts were compared to (1) the NCBI non-redundant nucleotide (nt) database with the use of Reciprocal BLASTn; and (2) the UniProt Swiss-Prot protein database with the use of Reciprocal BLASTx. Orthologs were identified if they were the symmetrical best hits in each reciprocal all-against-all (i.e., Reciprocal Best Hit) in the BLASTn and BLASTx search [28]. Briefly, orthologs to the sequences in the nt and Swiss-Prot databases were identified first by BLASTing the assembled transcript to the database. The highest-scoring hit was obtained and then BLASTed against the database of the assembled transcripts. The hit in the nt and Swiss-Prot databases was considered an ortholog of the assembled transcript if and only if the second BLAST returned the assembled transcript that was the highest scorer in the first BLAST.

As an alternative approach to annotate the assembled transcripts, protein-coding regions within the transcripts were first identified using the TransDecoder algorithm [23]. Briefly, 500 of the longest Open Reading Frames (ORFs) were extracted and used to build a Markov model based on hexamers. These likely coding sequences were randomized to provide a sequence composition corresponding to a non-coding sequence. All of the longest ORFs in each of the six possible reading frames were scored according to the Markov Model (log likelihood ratio based on coding/noncoding). If the proper coding frame of the putative ORF scored positive and was the highest of the other presumably wrong reading frames, then that ORF was reported. If a high-scoring ORF was eclipsed by (fully contained within the span of) a longer ORF in a different reading frame, it was excluded. The likely protein-coding regions were then subjected to (1) BLASTp searching against UniProtKB/Swiss-Prot with an e-value cut-off of $1.0 \times 10^{-6}$ [26,27], (2) a protein domain search via HMMScan, (3) transmembrane helicase prediction by TMHMM and (4) signal peptide prediction by SignalP.

\section{Discovery of tissue-enriched and 0 . melastigma-enriched genes}

An annotation-based approach was used to discover the tissue-enriched genes of O. melastigma. Quality-filtered 
transcripts with Reciprocal Best Hits (nt database and UniProt) were considered. For the brain and liver, of which both male and female transcriptomes were sequenced, matches to annotations were merged, and a union set was used. To compare the transcriptome between $O$. melastigma and its freshwater counterpart O. latipes, we obtained 2 independent sets of whole-fish, deep RNA-Seq data from the NCBI Sequence Read Archive (SRA) under Accession SRP004363 and SRP032993 and calculated the transcript expression based on our Reads-combined Assembly of the O. melastigma transcriptome. Briefly, $O$. melastigma transcripts with $\geq 8$ reads, but without any read-count in both independent freshwater RNASeq datasets were considered to be putative O. melastigmaenriched transcripts. O. melastigma-enriched transcripts across a dynamic range of expression were then subjected to $\mathrm{qPCR}$ validation to determine the optimal read-count threshold. Since the O. latipes RNA-Seq dataset we retrieved from NCBI SRA were yet to be published, we only sought to discover $O$. melastigma-enriched genes with respective to O. latipes.

\section{qPCR validation in independent samples}

Quantitative real-time PCR was used to detect the expression of select genes that are closely related to the functions of corresponding tissues, and $18 \mathrm{~S}$ ribosomal RNA (18S) was used as reference gene for qPCR normalization. The primer sequences are listed in Additional file 1: Table S1. cDNA was synthesized from $1 \mu \mathrm{g}$ of total RNA extracted from an independent set of medaka using the SuperScript ${ }^{\circ}$ VILO $^{\mathrm{mm}}$ cDNA Synthesis Kit (Life Technologies). The reverse transcription reactions were incubated in a C1000 Thermal Cycler (Bio-Rad) at $25^{\circ} \mathrm{C}$ for $10 \mathrm{~min}, 42^{\circ} \mathrm{C}$ for $60 \mathrm{~min}$ and $85^{\circ} \mathrm{C}$ for $5 \mathrm{~min}$ and then held at $4^{\circ} \mathrm{C}$. qRT-PCR was performed using the StepOnePlus Real-Time PCR system (Applied Biosystems). The 20- $\mu \mathrm{l}$ PCR reaction included $1 \mu \mathrm{l}$ of RT product, $10 \mu \mathrm{l}$ of KAPA SYBR ${ }^{\circ}$ FAST qPCR Master Mix (2X), $0.5 \mu$ of each primer $(10 \mu \mathrm{M})$, and $8 \mu \mathrm{l}$ of nuclease-free water. The reactions were incubated in a 96 -well optical plate at $95^{\circ} \mathrm{C}$ for $10 \mathrm{~min}$, followed by 40 cycles at $95^{\circ} \mathrm{C}$ for $15 \mathrm{sec}$ and $60^{\circ} \mathrm{C}$ for $1 \mathrm{~min}$. Reactions were run in triplicate and included a no-template control for each gene. The relative expression ratio of target/18S was calculated according to the method described by Pfaffl [29]:

$$
\begin{aligned}
\text { Expression ratio }= & \mathrm{E}_{\text {target }}{ }^{\mathrm{CP} \text { target }(\text { control-treatment })} \\
& / \mathrm{E}_{18 \mathrm{~S}}{ }^{\mathrm{CP} 18 \mathrm{~S} \text { (control-treatment) }}
\end{aligned}
$$

where $\mathrm{E}=10^{(-1 / \text { slope })}$ and $\mathrm{CP}$ is the crossing point at which fluorescence rises above background. Statistical significance was calculated using the Wilcoxon-MannWhitney test.

\section{Genome reference, genomic resources and tools used}

The medaka HdrR reference genome v.72.1 was retrieved from Ensembl [30], and the RNA-Seq data of freshwater O. latipes were retrieved from NCBI SRA (SRP004363 and SRP032993). STAR aligner [31] was used to align the transcriptome data to the genome, and reference mapping of the O. latipes UniGENE and RNA-Seq datasets to the assembled transcript re-mapping was performed using BWA-MEM v.0.7.5a-r405 and Novoalign v3.00.05 (http://www.novocraft.com/). Gene Ontology enrichment (Biological Process, Cellular Component, and Molecular Function) was performed using BinGO [32], which is implemented in Cytoscape (http://www.cytoscape.org/).

\section{Results and discussion}

Transcriptome sequencing of 4 organs (brain, liver, ovary and testis) of male and female O. melastigma in 6 libraries yielded $34.81 \mathrm{Gbp}$ of mRNA sequences from approximately 505 million $\sim 70$-bp paired-end reads (average 84 million reads per tissue). The coverage for each library was more than 100 -fold based on the transcriptome size of the freshwater counterpart $O$. latipes. A previous study suggested that such sequencing depth, coupled with stringent sequence reads quality filtering, is optimal for tissue specific transcriptome assembly [33]. Four hundred and twenty-two million quality-trimmed reads, corresponding to $28.5 \mathrm{Gbp}$ were subjected to downstream analysis. The sequencing statistics and technical details are shown in Additional file 2: Table S2.

\section{Comparison between the transcriptome of freshwater and marine medaka}

In order to estimate the divergence of the O. melastigma and O. latipes transcriptomes, we first assessed their average nucleotide identities at the transcript level. Based on the mRNA transcripts deposited in the NCBI nucleotide database, orthologs in O. melastigma and $O$. latipes were identified using Reciprocal BLAST. The reciprocal best hit (RBH) was found for 58.6\% (211/360) of the O. melastigma transcripts. Among the RBHs, the average identity was $91.5 \%$ (median: $91.8 \%$ ), suggesting an extensive diversity between the two species. (Figure 2A-B). In line with our observation, phylogeographic studies of medaka using allozymes and mitochondrial DNA sequences have revealed a genetic diversity in the Oryzias family [34-36]. The studies showed that wild populations of medaka were divided into four major regionally differentiated groups and the Nei's genetic distances among these groups are very large (0.35-0.88).

Using our RNA-Seq data, we then assessed if the Hd-rR (O. latipes) reference genome was suitable for marine counterparts, such as $O$. melastigma. For the two independent $O$. latipes RNA-Seq experiments, the majority of sequence reads (84.6-99.3\%) could be aligned onto the 


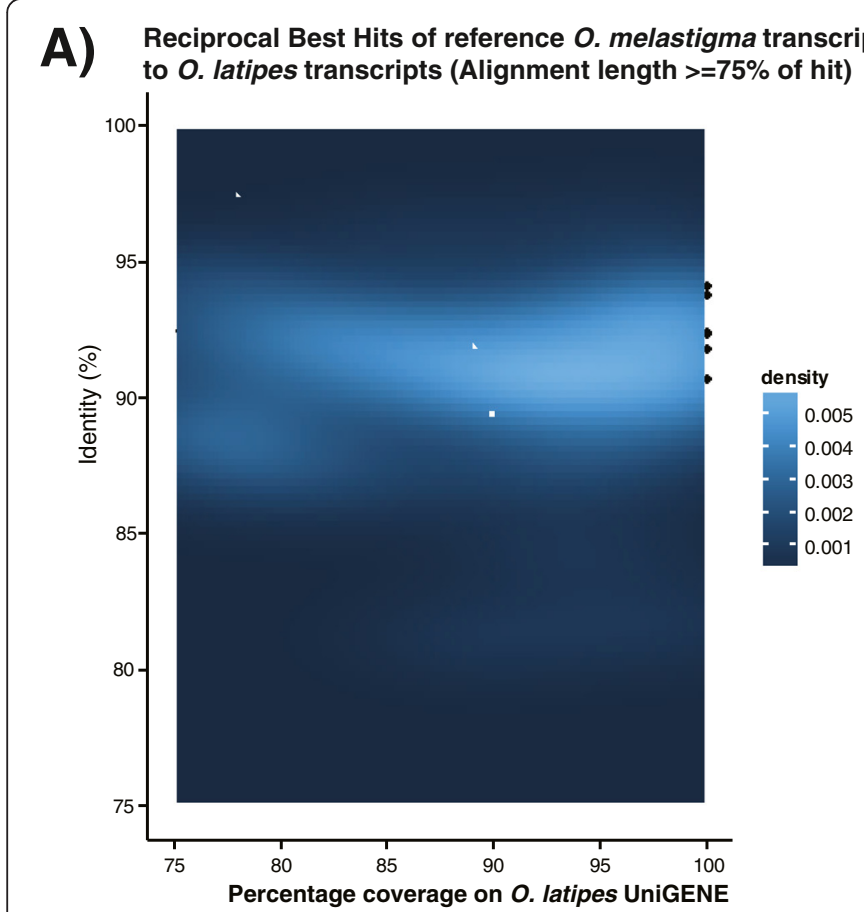

C) Percentage of mapping of O. melastigma RNA-Seq data to O. latipes UniGENE

- Mapped reads \% $\quad$ Properly mapped reads \%

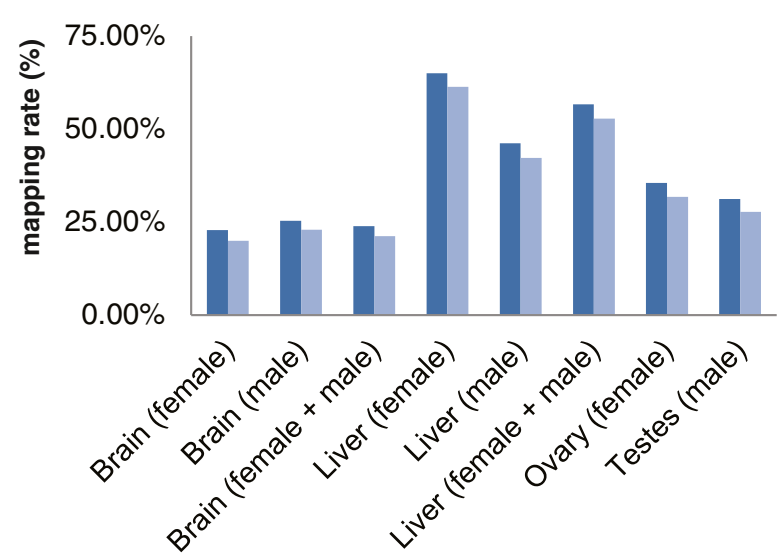

B) All Reciprocal Best Hits of reference O. melastigma transcript to $O$. latipes transcripts

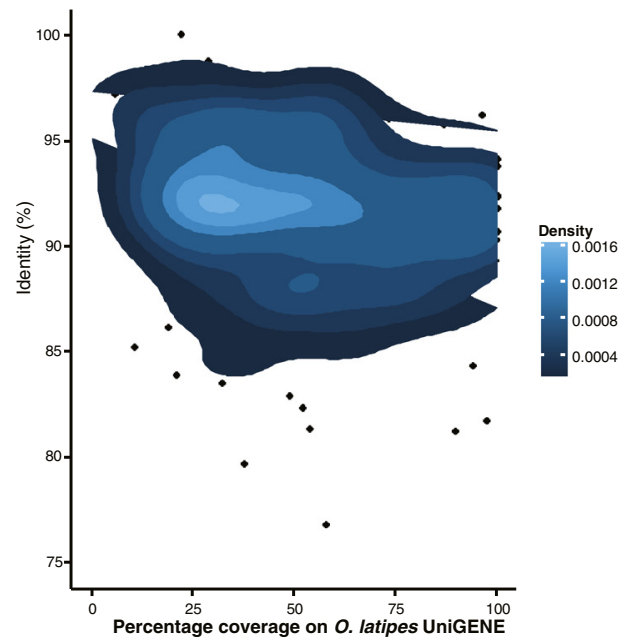
Matches of assembled transcripts to $O$. latipes
UniGENE

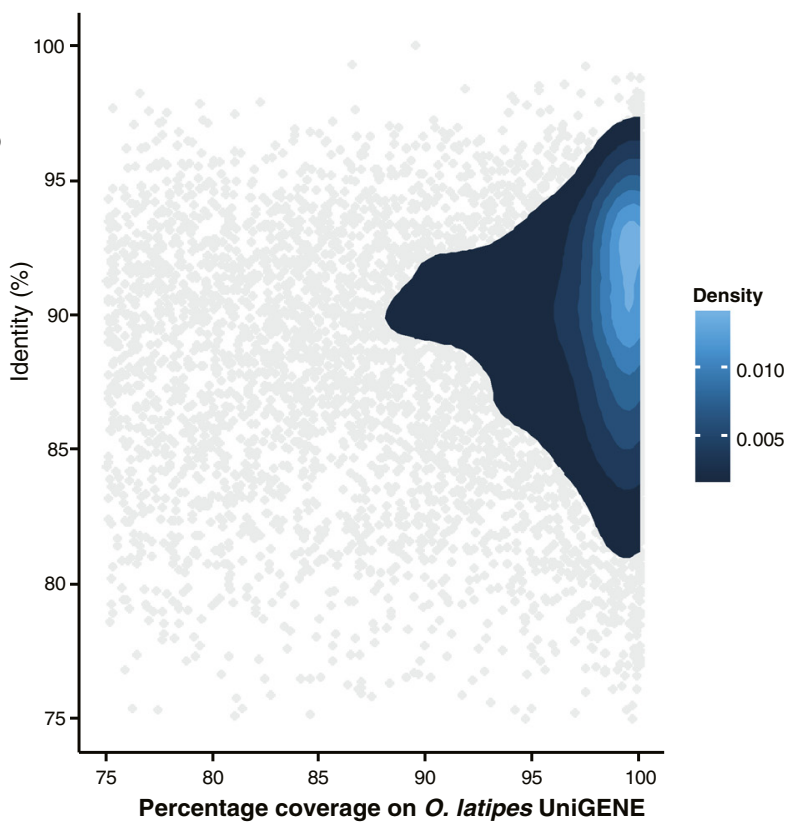

Figure 2 Transcriptome divergence between marine and freshwater medaka. A) Nucleotide identity between Oryzias melastigma and Oryzias latipes orthologs deposited in the NCBI nucleotide database. Only those with an alignment length $>75 \%$ are shown. B) Nucleotide identity between all $\mathrm{O}$. melastigma and $\mathrm{O}$. latipes orthologs. C) Low mapping rate of $\mathrm{O}$. melastigma RNA-Seq data generated in this study onto the $O$. latipes UniGENE dataset. The mapping rate is highest for liver and lowest for brain. D) The nucleotide identity between transcripts (Ensemble Assembly) assembled in this study and the O. latipes UniGENE dataset. Only those with alignment length $>75 \%$ are shown.

Hd-rR reference genome (mismatch rate: $0.43-0.48 \%$; unique aligned: 70.2-81.4\%). However, when the O. melastigma quality-trimmed reads were aligned to the Hd-rR genome, the mapping rate ranged from only 38.4 to $52.3 \%$ (mismatch rate: 4.6-5.7\%). Similarly, only a minority (22.8-65.0\%) of reads could be aligned onto the O. latipes UniGENE dataset, meaning that our $O$. melastigma RNA-Seq data comprises a significant portion of transcribed sequences that could not be unaligned and thus might be absent in the existing freshwater medaka genome and transcriptome sequences. Thus, the current $O$. latipes sequences might not be suitable for genome-wide expression studies of O. melastigma (Figure 2C and Additional file 3: Table S3).

Our observations were not surprising and were in fact in line with previous finding suggesting that within the $O$. latipes species, the genome-wide SNP rate between 
the Hd-rR and HNI strains is highest (3.42\%) among vertebrate species [15]. Such high divergence among different medaka species re-iterates that a marine transcriptome reference dataset, such as the emerging marine model O. melastigma, is imperative for studies that assess the responses of marine species to pollutants, toxins and stresses at the molecular level.

\section{Transcriptome assembly and generation of a consensus transcriptome}

De novo assemblies of each library using Trinity resulted in an average of 85098 (51533-132296) contigs per sample. Brain tissue had the highest contig count, totaling approximately $15.6 \mathrm{Mbp}$. The lowest contig count was observed in liver tissue, with approximately $4.6 \mathrm{Mbp}$. The average contig length was $1106 \mathrm{bp}$ and the contig N50 was 2162 bp. Nearly all transcripts (99.93\%) had a coverage greater than 1 and were subjected to downstream annotation (Additional file 4: Table S4).

Using the transcriptome assemblies, we sought to rule out the possibility that the previously observed lowmapping rate of the O. melastigma RNA-Seq data onto the O. latipes UniGENE dataset was due to aligner bias. We assessed the recovery of the O. latipes UniGENE dataset based on our assembly result using BLASTn. With an e-value threshold of $1.0 \times 10^{-6}[26,27]$, we found that, at most, $72.3 \%$ of the O. latipes UniGENE dataset $(45.6 \%$ if $\geq 70 \%$ of the UniGENE dataset must be covered in terms of transcript length) could be matched to our assemblies, with an average identity, in bases, between transcripts of the two species of $89.6 \%$ (Figure 2D and Additional file 5: Table S5). In other words, the mismatch rate was again approximately $10 \%$, suggesting that $O$. melastigma might be divergent from O. latipes.

The core eukaryotic genes (CEGs) [25] are highly conserved, present in all eukaryotic species and found in low numbers of in-paralogs in different species. A majority of the CEGs are expected to be present in a quality transcriptome assembly. Among the 248 CEGs, 99.6\% were recovered in the "Reads-combined Assembly" (see below), and the average e-value was highly significant (2.05E-14) and average percentage identity of the matched transcripts were $95.9 \%$ (details in Additional file 6: Table S6).

When comparing the assembled transcripts with known O. melastigma mRNA sequences using BLASTn and known O. melastigma protein sequences using BLASTx, we found that $92.4 \%$ (327/354) of known transcripts were recovered in the Reads-combined Assembly, while 86.4\% (323/374) of the known O. melastigma protein sequences were recovered, suggesting our assembly should be largely complete. However, some tissue-enriched genes in organs other than the brain, liver, ovary and testis may have been missed (details in Additional file 7: Table S7, Additional file 8: Table S8, Additional file 9: Table S9).

To aid in the comparison of gene expression among different $O$. melastigma tissues, we explored two approaches to generate a single consensus transcriptome assembly; (1) Reads-combined Assembly: sequence reads for all tissues were combined prior to being subjected to de novo assembly [23] and (2) Contigs-clustered Assembly: assembly was performed individually for each library. Redundant transcripts were identified, and representative transcripts were chosen by clustering $[21,37]$. When comparing the two approaches, the Reads-combined Assembly recovered more CEGs than the Contigs-clustered Assembly. More importantly, the Reads-combined Assembly had significantly more RBHs than the Contigs-clustered Assembly (14,628 vs 12,145$)$. Moreover, the average contig length (1302 bp vs $1086 \mathrm{bp}$ ) and N50 (2908 bp vs 2450 bp) was longer for the Readscombined Assembly. Taken together, we believe the Readscombined Assembly represents a more complete consensus transcriptome assembly for inter-organ comparison.

\section{Protein-coding genes expression in the brain, liver, ovary} and testis of $\mathrm{O}$. melastigma

Protein-coding ORF prediction followed by Reciprocal Best Hit BLAST resulted in 14,628 annotated genes that were found across the brain, liver and gonadal tissues of O. melastigma (Table 1). The highest numbers of annotated genes were expressed in brain tissue. In females, 14240, 9200 and 13240 annotated genes were identified in brain, liver and ovary, respectively. In males, 13,796, 10,763 , and 13,618 annotated genes were identified in the brain, liver and testis, respectively. For brain tissue, the female- and male- combined assembly improved the assembly slightly and yielded 14,267 annotated genes. For liver tissue, the female- and male- combined assembly significantly improved the discovery and resulted in 11,438 annotated genes.

\section{Tissue-enriched genes in 0. melastigma}

Global comparison of annotated genes showed that 7157 $(34.5 \%)$ genes that were annotated in only a single tissue.

Table 1 Number of genes identified in different organs of O. melastigma

\begin{tabular}{llc}
\hline Organ & Gender & Number of identified genes \\
\hline Brain & Female & 14,240 \\
& Male & 13,796 \\
Liver & Female & 9,200 \\
& Male & 10,763 \\
Ovary & Female & 13,240 \\
Testis & Male & 13,618 \\
Total number & $/$ & 14,628 \\
\hline
\end{tabular}


We found 2692 brain-enriched genes, while 2848 genes were liver-enriched, and 2007 genes were gonad-enriched. Furthermore, 6821 annotated genes were common to all tissues in both males and females (Figure 3). The gonadenriched genes were enriched in the following GO terms: sexual reproduction and gamete generation. The brainenriched genes were enriched in functions related to channel activity, synaptic transmission and cell-cell adhesion. The liver-enriched genes were enriched in functions related to metabolic processes, transferase and mannosidase activity (Table 2, Additional file 10: Table S10).

We identified the tissue-enriched genes using a more conservative read-count approach. The expression of tissue-enriched genes was validated using qPCR analysis. Some of the genes were closely related to the functions of corresponding tissues. Our results demonstrated that gap junction beta-1 protein (CXB1) and potassium voltage-gated channel subfamily A member 2 (KCNA2) were highly expressed in both male and female marine medaka brain tissue (Figure 4A). Gap junction protein is the major component of gap junction channels that controls the exchange of ions and small molecules between cells. In the human brain, $C X B 1$ is highly expressed in neurons and oligodendrocytes and appears to be critical for the functions of Schwann cells, which are responsible for the myelination of nerves in the peripheral nervous system [38,39]. KCNA2 is present in most voltage-gated ion channels and plays important biological functions in the brain, including neurotransmitter release and neuronal excitability. Knockdown of KCNA2 reduces the total voltage-gated potassium current, resulting in increased

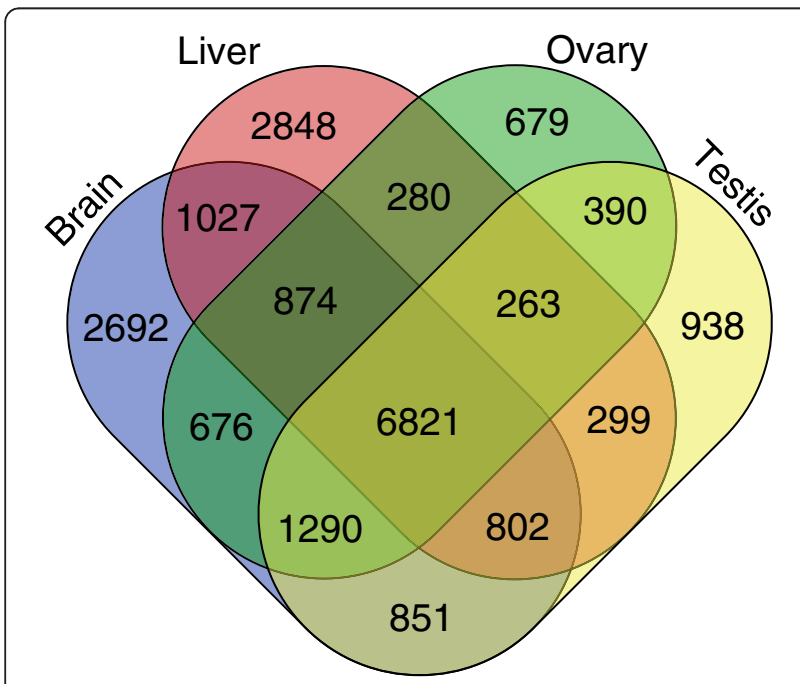

Figure 3 Global comparison of annotated genes in the brain, liver, ovary and testis of marine medaka. Of the identified genes, 2692 were brain-enriched. 2848 genes were liver-enriched, and 2007 genes were gonad-enriched. 6821 annotated genes were common to all tissues in both males and females. excitability in neurons and neuropathic pain symptoms in rats [40]. The identification of genes related to brain functions could largely facilitate the use of marine medaka as an in vivo model for neuro-toxicological studies.

Our qPCR analysis also indicated high expression of ammonium transporter Rh type $\mathrm{B}(R h B G)$ and bone morphogenetic protein 10 (BMP10) in the marine medaka liver (Figure 4B). Studies in mice have demonstrated that RhBG is highly expressed in the perivenous hepatocytes of the liver, which is an important tissue for ammonium metabolism and mediates ammonium uptake $[10,12]$. BMP10 is a member of the transforming growth factor $\beta$ (TGF $\beta$ ) superfamily, whose members interact with membrane-bound receptors to exert their biological functions [41]. Analysis of BMP10-deficient mice demonstrated that $B M P 10$ has an exclusive function in early cardiac development [42]. However, its function in the liver is still elusive. We also found an elevated level of methyltransferase-like 21C (MTL21C) and forkhead box protein $\mathrm{N} 5$ (FOXN5) in the ovaries of marine medaka (Figure 4C). MTL21C is a newly identified lysine methyltransferase that regulates the activities of various molecular chaperones, as well as the lysine residues in heat shock protein 70 [43]. Studies in pigs have demonstrated that heat shock chaperones play an important role in thermal stress adaptation [44]. FOXN5 is Forkhead-box (FOX) gene which is implicated in embryogenesis through transcriptional regulation. Study in mouse demonstrated that germ-line mutation of FOXN5 gene in the mouse lineage might lead to divergent scenario of early embryogenesis through the deregulation of FOXN5 target genes in mouse early embryos $[45,46]$. Last, our result demonstrated that succinate receptor 1 (SUCR1) and the Ig-like V-type domain-containing protein FAM187A (FAM187A) were highly expressed in marine medaka testicular tissues (Figure 4D). In humans, SUCR1 is expressed in a variety of tissues, including adipose, liver, and kidney tissue [47]. This protein is a $\mathrm{G}$ protein-coupled receptor that senses cellular stresses such as hypoxia, toxicity, and hyperglycemia. Taken together, our results identified a number of tissue-enriched genes in the brain, liver, testis and ovary of marine medaka and may largely facilitate the use of $O$. melastigma for marine ecotoxicological studies at the organ level.

\section{Marine-to-freshwater orthologous transcripts and marine-enriched transcripts}

To compared the conservativeness between marine medaka (O. melastigma) and freshwater medaka (O. latipes), Reciprocal Best Hit BLASTn was used. We estimated $O$. melastigma and O. latipes had 5880 orthologous protein-coding transcripts, requiring more than $70 \%$ length recovery of $O$. latipes transcripts (Additional file 11: Table S11). 
Table 2 Functional enrichment of Gene Ontology terms in organ-enriched genes

\begin{tabular}{|c|c|c|c|c|}
\hline & Gene Ontology & ID & Category & $\begin{array}{l}\text { Benjamini \& Hochberg } \\
\text { corrected p-value }\end{array}$ \\
\hline \multirow{2}{*}{$\begin{array}{l}\text { Gonad-enriched genes common to } \\
\text { male and female marine medaka }\end{array}$} & sexual reproduction & GO:0019953 & Biological Process & 4.42E-04 \\
\hline & gamete generation & GO:0007276 & Biological Process & 4.42E-04 \\
\hline \multirow[t]{7}{*}{ Brain-enriched genes } & gated channel activity & GO:0022836 & Molecular Function & $8.81 \mathrm{E}-10$ \\
\hline & signaling & GO:0023052 & Biological Process & $2.98 \mathrm{E}-06$ \\
\hline & transmission of nerve impulse & GO:0019226 & Biological Process & 8.35E-06 \\
\hline & potassium channel activity & GO:0005267 & Molecular Function & 8.80E-06 \\
\hline & cell-cell adhesion & GO:0098609 & Biological Process & 4.92E-05 \\
\hline & nervous system development & GO:0007399 & Biological Process & 4.20E-05 \\
\hline & synapse & GO:0045202 & Cellular Component & $2.25 \mathrm{E}-04$ \\
\hline \multirow[t]{7}{*}{ Liver-enriched genes } & cellular macromolecule metabolic process & GO:0044260 & Biological Process & 1.57E-12 \\
\hline & RNA metabolic process & GO:0016070 & Biological Process & 3.34E-07 \\
\hline & nitrogen compound metabolic process & GO:0006807 & Biological Process & 4.62E-07 \\
\hline & transferase activity & GO:0016740 & Molecular Function & 1.09E-05 \\
\hline & protein modification process & GO:0036211 & Biological Process & 2.77E-03 \\
\hline & kinase activity & GO:0016301 & Molecular Function & 2.87E-03 \\
\hline & mannosidase activity & GO:0015923 & Molecular Function & 1.10E-02 \\
\hline
\end{tabular}

The capability of animal cells to maintain a constant cell volume is prerequisite for cellular life. When eukaryotic cells are exposed to extracellular osmotic stress, they undergo rapid regulatory processes to maintain their cellular homeostatic status. The mechanism is particularly important in gill epithelia in fishes. Here, we showed the RNA-seq data from two medaka fishes that live in different osmotic environments. O. melastigma inhabits in brackish-water or fresh water around Begal Bay and Malay Peninsula; while O. latipes are found in fresh water of Japan, Korea and China. They encounter different osmotic environments and have been shown to have different osmotic tolerances in fresh water to seawater transfer experiments [48]. In a molecular point of view, the two fishes should have different osmoregulatory mechanisms. In fish biology, we know that the gill is the first osmoregulatory tissue to sense and response the osmotic challenges [49]. In addition, kidney and intestine play osmoregulatory roles in fish [50,51]. Although our transcriptome data of O. melastigma do not include the osmoregulartory tissues/organs, our data have identified several critical seawater acclimating ion transporters, such as cystic fibrosis transmembrane conductance regulator, sodium/potassium/chloride co-transporter, and sodium pump $\alpha$ and $\beta$. These ion transporters have been shown to be highly expressed in gills of SW acclimated fishes, such as eel, and tilapia [52-54]. The identification of these ion transporters in the O. melastigma suggested the possible use of our RNA-seq data for future osmoregulatory studies.
Furthermore, by using read-count approach and qPCR validation, we estimated that a lower boundary of 255 genes being only expressed in O. melastigma compared to those in the O. latipes database (Additional file 12: Table S12). The highly expressed genes in O. melastigma and some selected genes that might be functionally related to seawater adaptation were further validated by RT-PCR. Indeed, our results showed that a number of genes were highly expressed in $O$. melastigma but undetected in $O$. latipes (Figure 5). One of the O. melastigma-enriched genes, solute carrier and organic anion transporter (SO3A1), is commonly found in human brain tissue and epidermal keratinocytes. SO3A1 may play a role in the exchange of anions between cells, thus facilitating seawater adaptation [55]. In addition, it mediates the transport of thyroxine and vasopressin [56] that is important in osmoregulation [57,58]. Similarly, another solute carrier, solute carrier family 12 member 5 (S12A5), is commonly found in brain. It is a potassium-chloride co-transporter, which is highly expressed in neurons [59]. In addition, the sodium-calcium-potassium exchanger 2 (NCKX2) is a polytopic membrane protein that drives $\mathrm{Ca}^{2}+$ extrusion across the plasma membrane [60]. All these three transporters mentioned above are highly expressed in the brain region. However, they all cannot be aligned in the recent existing freshwater medaka. Additionally, cardiac channels such as potassium voltage-gated channel subfamily D member 2 (KCND2) and plakophilin-2 (PKP2) also only be found in the marine medaka. KCND2 is critical in repolarizing the cardiac action potential [61], while $P K P 2$ is essential 


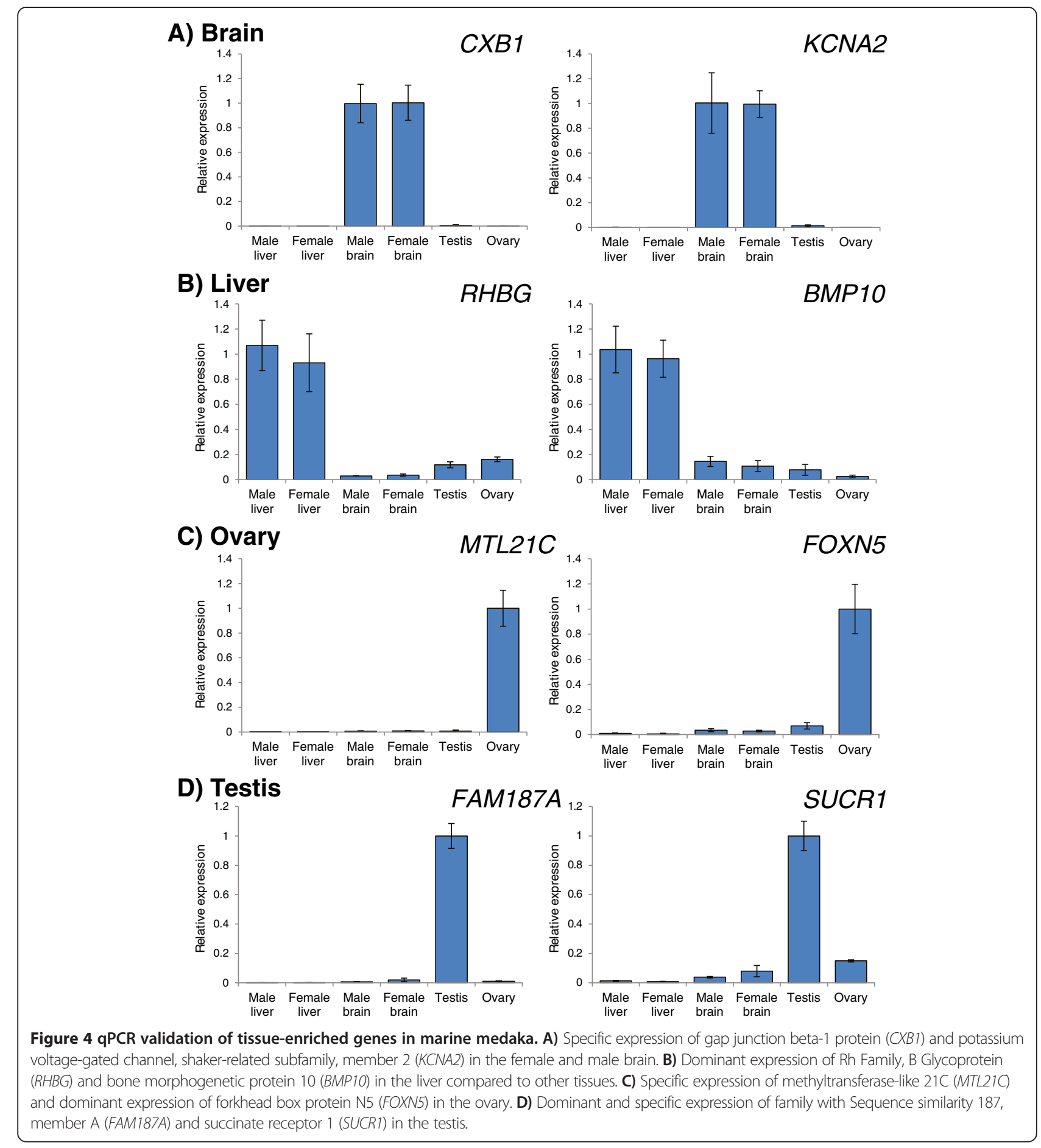

protein for building up of desmosome. PKP2 has been reported to be functionally related to sodium channel, and decreased in PKP2 expression leaded to downregulation of sodium current in cardiomyocytes of human $[62,63]$. The data presented here, hence provides opportunities for researchers to understand the ion transporters mechanism between two species by using our database as nucleotide references for different molecular probes.
Nevertheless, instead of using the existing model organism genome database, our findings suggest that researchers generate their own model transcriptome database for a more confident result. Even in the two close species we examined here, there are genes that cannot be aligned in the seawater medaka. It should be noted that some seawaterunique genes we mentioned above are common in different species; the reason that we cannot identify them in the 


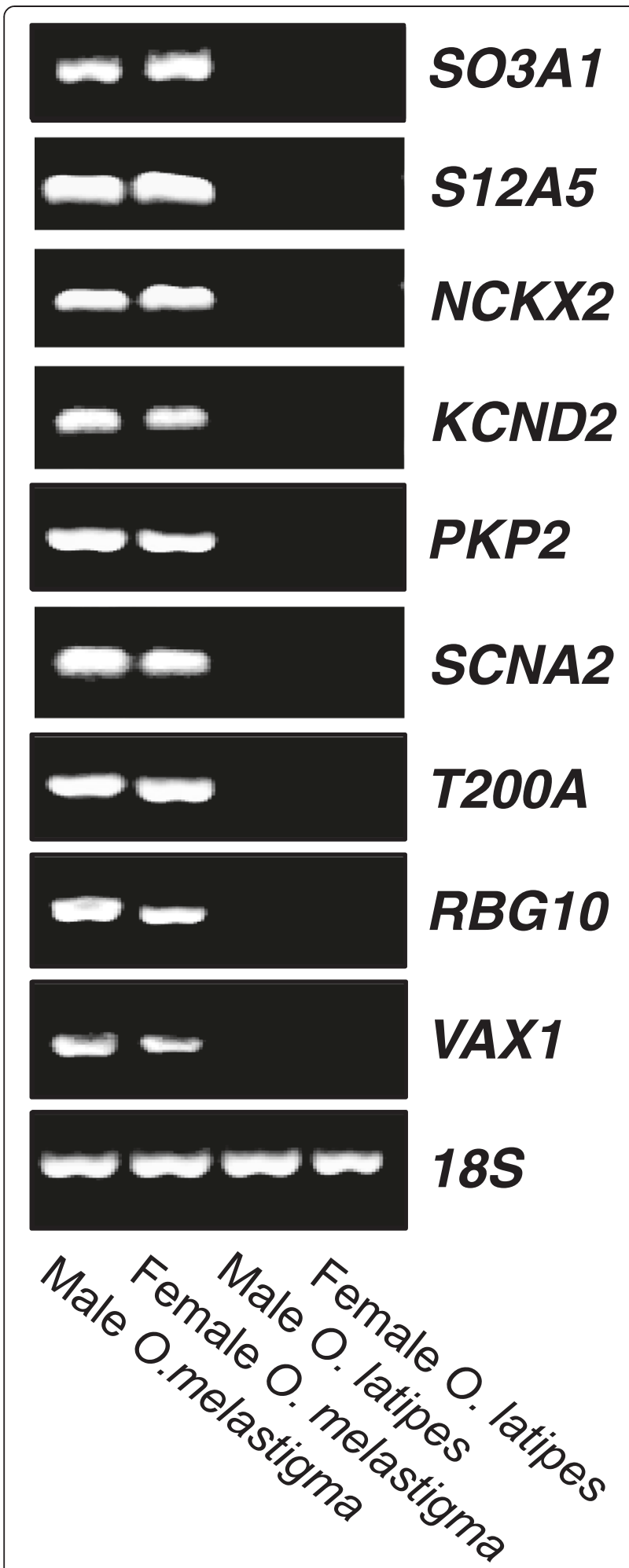

Figure 5 Validation of 0 . melastigma-enriched genes by RT-PCR. The marine medaka specific genes were validated in both male and female marine medaka against freshwater medaka. freshwater medaka may due to their low similarities between the two species. In fact, the ambient conditions, age, and the physiological state when tissue samples were collected influences the transcription rate of a gene, and whether or not a gene is expressed at all. We also note that allelic variation might explain the observed large divergence between the orthologous transcripts between marine and freshwater medaka. Nevertheless, this further supports the necessity of generating species-specific database for ecotoxicological studies.

\section{Conclusion}

This study provides a specific marine medaka transcriptome resource to the community that could facilitate future works on the marine medaka. We annotated more than 14,000 transcripts across four tissues in marine medaka and found 5880 orthologous transcripts between O. melastigma and O. latipes. Moreover, numerous tissue/organ-enriched genes were identified. Most importantly, we further investigated the possible divergence between $O$. melastigma and O. latipes, which suggests the importance of generating the model's own transcriptome database. This sequencing effort generated a valuable resource of coding DNA for a non-model species that will aid future studies assessing in vivo molecular responses to environmental stresses and biological toxicity in the marine environment.

\section{Availability of supporting data}

The sequence data from this study have been submitted to the NCBI Sequence Read Archive (SRA) (http://www.ncbi. nlm.nih.gov/sra) under the accession number SRP041838. The assembled transcripts were deposited in at DDBJ/ EMBL/GenBank under the accession GBFY00000000; GBFX00000000; GBFW00000000; GBFV00000000; GBFU 00000000; GBFT00000000; GBFS00000000; GBFR00000 000; GBFQ00000000 and GBGE00000000 under BioProject ID: 246584.

\section{Additional files}

Additional file 1: Table S1. $\mathrm{qPCR}$ Primer list. Primer list used in $\mathrm{QPCR}$ validation.

Additional file 2: Table S2. Sequencing summary. Summary of Illumina high-throughput sequencing.

Additional file 3: Table S3. Mapping statistics for freshwater medaka. Reference mapping of the $\mathrm{O}$. melastigma RNA-Seq data generated in this study onto the freshwater medaka genome and UniGENE database.

Additional file 4: Table S4. Transcriptome de novo assembly statistics. Technical statistics of the de novo transcriptome assembly.

Additional file 5: Table S5. Identity between the Oryzias latipes UniGENE dataset and Oryzias melastigma assembled transcripts.

Comparison between the O. latipes UniGENE dataset and O. melastigma assembled transcripts reveals divergent transcriptomes in terms of nucleotide identity. 
Additional file 6: Table S6. Recovery of eukaryotic core genes in the assembled transcriptome. Recovery of the 248 eukaryotic core genes in the assembled transcriptome.

Additional file 7: Table S7. Summary of the recovery of known 0 . melastigma sequences in the assembled transcriptome. Summary of the recovery of known $O$. melastigma sequences in the assembled transcriptome.

Additional file 8: Table S8. Details of the recovery of known O. melastigma mRNA sequences in the assembled transcriptome. Details of the recovery of known O. melastigma mRNA sequences in the assembled transcriptome.

Additional file 9: Table S9. Details of the recovery of known O. melastigma protein sequences in the assembled transcriptome. Details of the recovery of known O. melastigma protein sequences in the assembled transcriptome.

Additional file 10: Table S10. Gene Ontology enrichment for the tissue-enriched genes. Gene Ontology enrichment for the brain-, liverand gonad-enriched genes.

Additional file 11: Table S11. Orthologus transcripts between $O$. melastigma and $O$. latipes.

Additional file 12: Table S12. O. melastigma-enriched transcripts. Marine medaka O. melastigma transcripts that are not expressed in freshwater medaka O. latipes.

\section{Abbreviations}

(O. melastigma): Oryzias melastigma; (O. latipes): Oryzias latipes; (ORFs): Open Reading Frames; (RBH): Reciprocal Best Hit; (CEG): Core Eukaryotic Genes; (SRA): Sequence Read Archive.

\section{Competing interests}

The authors declare that they have no competing interests.

\section{Authors' contributions}

KPL participated in molecular experiments and drafted the manuscript, JWL carried out the transcriptome data analysis and drafted the manuscript, AT carried out the real time PCR analysis, KL and SYW participated in samples collection and samples preparation for transcriptome sequencing, SL carried out the transcriptome sequencing, WKFT, CKCW and TFC participated in the design of the study and performed the statistical analysis. DWTA and JMYC conceived of the study, RYCK and RSSW participated in experimental design and coordination and helped to draft the manuscript. KPL and JWL contributed equally in this work. All authors read and approved the final manuscript.

\section{Acknowledgements}

The authors thank Professor David Hinton for gifting the freshwater medaka Oryzias latipes. This work is supported by the State Key Laboratory in Marine Pollution (SKLMP) Seed Collaborative Research Fund (PJ: SKLMP/SCRF/0013) and Seed Funding Programme for Basic Research (HKU/SFPBR/201308159001). JWL is supported partly by a General Research Fund (GRF461712) to TFC.

\section{Author details}

'School of Biological Sciences, Kadoorie Biological Sciences Building, The University of Hong Kong, Pokfulam Road, Hong Kong, SAR, China. ${ }^{2}$ School of Life Sciences, Hong Kong Bioinformatics Centre, The Chinese University of Hong Kong, Hong Kong, SAR, China. ${ }^{3}$ Genome Research Centre, The Hong Kong Jockey Club Building for Interdisciplinary Research, The University of Hong Kong, 5 Sassoon Road, Pokfulam, Hong Kong, SAR, China. ${ }^{4}$ Department of Biology and Chemistry, City University of Hong Kong, Tat Chee Avenue, Kowloon, Hong Kong, SAR, China. ${ }^{5}$ Department of Biology, Hong Kong Baptist University, Hong Kong, SAR, China. ${ }^{6}$ The State Key Laboratory in Marine Pollution, Hong Kong, China.

Received: 4 June 2014 Accepted: 6 February 2015

Published online: 27 February 2015

\section{References}

1. Kong RY, Giesy JP, Wu RS, Chen EX, Chiang MW, Lim PL, et al. Development of a marine fish model for studying in vivo molecular responses in ecotoxicology. Aquat Toxicol. 2008;86(2):131-41.
2. Shi X, Du Y, Lam PK, Wu RS, Zhou B. Developmental toxicity and alteration of gene expression in zebrafish embryos exposed to PFOS. Toxicol Appl Pharmacol. 2008;230(1):23-32

3. Wu X, Huang Q, Fang C, Ye T, Qiu L, Dong S. PFOS induced precocious hatching of Oryzias melastigma-from molecular level to individual level. Chemosphere. 2012;87(7):703-8.

4. Wheeler JR, Leung KM, Morritt D, Sorokin N, Rogers H, Toy R, et al. Freshwater to saltwater toxicity extrapolation using species sensitivity distributions. Environ Toxicol Chemistry / SETAC. 2002;21(11):2459-67.

5. Dong S, Kang M, Wu X, Ye T. Development of a Promising Fish Model (Oryzias melastigma) for Assessing Multiple Responses to Stresses in the Marine Environment. BioMed Res Int. 2014;2014:563131.

6. Chen X, Li L, Cheng J, Chan LL, Wang DZ, Wang KJ, et al. Molecular staging of marine medaka: a model organism for marine ecotoxicity study. Mar Pollut Bull. 2011:63(5-12):309-17.

7. Wittbrodt J, Shima A, Schartl M. Medaka-a model organism from the far East. Nat Rev Genet. 2002;3(1):53-64

8. Rhee JS, Kim BM, Choi BS, Choi IY, Wu RS, Nelson DR, et al. Whole spectrum of cytochrome P450 genes and molecular responses to water-accommodated fractions exposure in the marine medaka. Environ Science Technol. 2013:47(9):4804-12

9. Wang $M$, Wang $Y$, Zhang L, Wang J, Hong H, Wang D. Quantitative proteomic analysis reveals the mode-of-action for chronic mercury hepatotoxicity to marine medaka (Oryzias melastigma). Aquat Toxicol. 2013;130-131:123-31.

10. Ye RR, Lei EN, Lam MH, Chan AK, Bo J, van de Merwe JP, et al. Gender-specific modulation of immune system complement gene expression in marine medaka Oryzias melastigma following dietary exposure of BDE-47. Environ Sci Pollut Res Int. 2011;19(7):2477-87.

11. Huang Q, Fang C, Wu X, Fan J, Dong S. Perfluorooctane sulfonate impairs the cardiac development of a marine medaka (Oryzias melastigma). Aquat Toxicol. 2011;105(1-2):71-7.

12. Tian L, Wang M, Li X, Lam PK, Wang M, Wang $D$, et al. Proteomic modification in gills and brains of medaka fish (Oryzias melastigma) after exposure to a sodium channel activator neurotoxin, brevetoxin-1. Aquat Toxicol. 2011;104(3-4):211-7.

13. Hwang DS, Kim BM, Au DW, Lee JS. Complete mitochondrial genome of the marine medaka Oryzias melastigma (Beloniformes, Adrianichthyidae) Mitochondrial DNA. 2012:23(4):308-9.

14. Bo J, Cai L, Xu JH, Wang KJ, Au DW. The marine medaka Oryzias melastigma-a potential marine fish model for innate immune study. Mar Pollut Bull. 2011:63(5-12):267-76

15. Kasahara M, Naruse K, Sasaki S, Nakatani Y, Qu W, Ahsan B, et al. The medaka draft genome and insights into vertebrate genome evolution. Nature. 2007:447(7145):714-9.

16. Takehana $Y$, Naruse K, Sakaizumi M. Molecular phylogeny of the medaka fishes genus Oryzias (Beloniformes: Adrianichthyidae) based on nuclear and mitochondrial DNA sequences. Mol Phylogenet Evol. 2005;36(2):417-28.

17. M. K, Murata K, Naruse K, Tanaka M. Medaka: Biology, Management, and Experimental protocols, Wiley-Blackwell, lowa, USA 2009.

18. Li H, Durbin R. Fast and accurate short read alignment with BurrowsWheeler transform. Bioinformatics. 2009;25(14):1754-60.

19. Grabherr MG, Haas BJ, Yassour M, Levin JZ, Thompson DA, Amit I, et al. Full-length transcriptome assembly from RNA-Seq data without a reference genome. Nat Biotechnol. 2011;29(7):644-52

20. Zhao QY, Wang Y, Kong YM, Luo D, Li X, Hao P. Optimizing de novo transcriptome assembly from short-read RNA-Seq data: a comparative study. BMC Bioinformatics. 2011;12 Suppl 14:S2.

21. Fu L, Niu B, Zhu Z, Wu S, Li W. CD-HIT: accelerated for clustering the next-generation sequencing data. Bioinformatics. 2012;28(23):3150-2.

22. Duan J, Xia C, Zhao G, Jia J, Kong X. Optimizing de novo common wheat transcriptome assembly using short-read RNA-Seq data. BMC Genomics. 2012;13:392

23. Haas BJ, Papanicolaou A, Yassour M, Grabherr M, Blood PD, Bowden J, et al. De novo transcript sequence reconstruction from RNA-seq using the Trinity platform for reference generation and analysis. Nat Protoc. 2013;8(8):1494-512.

24. O'Neil ST, Emrich SJ. Assessing De Novo Transcriptome Assembly Metrics for Consistency and Utility. BMC Genomics. 2013;14(1):465

25. Parra G, Bradnam K, Korf I. CEGMA: a pipeline to accurately annotate core genes in eukaryotic genomes. Bioinformatics. 2007;23(9):1061-7. 
26. Ewen-Campen B, Shaner N, Panfilio KA, Suzuki Y, Roth S, Extavour CG. The maternal and early embryonic transcriptome of the milkweed bug Oncopeltus fasciatus. BMC Genomics. 2011;12:61

27. Du H, Bao Z, Hou R, Wang S, Su H, Yan J, et al. Transcriptome sequencing and characterization for the sea cucumber Apostichopus japonicus (Selenka, 1867). PLOS ONE. 2012:7(3):e33311.

28. Rivera MC, Jain R, Moore JE, Lake JA. Genomic evidence for two functionally distinct gene classes. Proc Natl Acad Sci U S A. 1998;95(11):6239-44.

29. Pfaffl MW. A new mathematical model for relative quantification in real-time RT-PCR. Nucleic Acids Res. 2001;29(9):e45.

30. Flicek P, Amode MR, Barrell D, Beal K, Billis K, Brent S, et al. Ensembl 2014. Nucleic Acids Res. 2014;42(Database issue):D749-55.

31. Dobin A, Davis CA, Schlesinger F, Drenkow J, Zaleski C, Jha S, et al. STAR: ultrafast universal RNA-seq aligner. Bioinformatics. 2013;29(1):15-21.

32. Maere S, Heymans K, Kuiper M. BiNGO: a Cytoscape plugin to assess overrepresentation of gene ontology categories in biological networks. Bioinformatics. 2005:21(16):3448-9.

33. Francis WR, Christianson LM, Kiko R, Powers ML, Shaner NC, SH DH. A comparison across non-model animals suggests an optimal sequencing depth for de novo transcriptome assembly. BMC Genomics. 2013;14:167.

34. Sakaizumi M, Moriwaki K, Egami N. Allozymic variation and regional differentiation in wild population of the fish Oryzias latipes. Copeia. 1983:1983(2):311-8.

35. Sakaizumi M. Genetic divergence in wile populations of the Medaka Oryzias latipes (Pisces: Oryziatidae) from Japan and China. Genetica. 1986:69:119-25.

36. Matsuda M, Yonekawa H, Hamaguchi S, Sakaizumi M. Geographic variation and diversity in the mitochondrial DNA of the medaka, Oryzias latipes, as determined by restriction endonuclease analysis. Zool Sci. 1997;17:517-26.

37. Fu B, He S. Transcriptome analysis of silver carp (Hypophthalmichthys molitrix) by paired-end RNA sequencing. DNA Res. 2012;19(2):131-42.

38. Aronica E, Gorter JA, Jansen GH, Leenstra S, Yankaya B, Troost D. Expression of connexin 43 and connexin 32 gap-junction proteins in epilepsy-associated brain tumors and in the perilesional epileptic cortex. Acta Neuropathol. 2001;101(5):449-59.

39. Tang C, Zelenak C, Volkl J, Eichenmuller M, Regel I, Frohlich H, et al. Hydration-sensitive gene expression in brain. Cell Physiol Biochem. 2011;27(6):757-68.

40. Zhao X, Tang Z, Zhang H, Atianjoh FE, Zhao JY, Liang L, et al. A long noncoding RNA contributes to neuropathic pain by silencing Kcna2 in primary afferent neurons. Nat Neurosci. 2013;16(8):1024-31.

41. Wu M, Chen W, Mi J, Chen D, Wang W, Gao H. Expression analysis of BMP2, BMP5, BMP10 in human colon tissues from Hirschsprung disease patients. Int J Clin Exp Pathol. 2014;7(2):529-36.

42. Chen H, Brady Ridgway J, Sai T, Lai J, Warming S, Chen H, et al. Contextdependent signaling defines roles of BMP9 and BMP10 in embryonic and postnatal development. Proc Natl Acad Sci U S A. 2013;110(29):11887-92.

43. Cloutier $\mathrm{P}$, Lavallee-Adam M, Faubert D, Blanchette M, Coulombe B. A newly uncovered group of distantly related lysine methyltransferases preferentially interact with molecular chaperones to regulate their activity. PLoS Genet. 2013;9(1):e1003210.

44. Pennarossa G, Maffei S, Rahman MM, Berruti G, Brevini TA, Gandolfi F. Characterization of the constitutive pig ovary heat shock chaperone machinery and its response to acute thermal stress or to seasonal variations. Biol Reprod. 2012;87(5):119.

45. Katoh M, Katoh M. Germ-line mutation of Foxn5 gene in mouse lineage. Int J Mol Med. 2004;14(3):463-7.

46. Katoh M, Katoh M. Identification and characterization of human FOXN5 and rat Foxn5 genes in silico. Int J Oncol. 2004;24(5):1339-44.

47. Ariza AC, Deen PM, Robben JH. The succinate receptor as a novel therapeutic target for oxidative and metabolic stress-related conditions. Front Endocrinol. 2012;3:22

48. Inoue K, Takei Y. Diverse adaptability in oryzias species to high environmental salinity. Zool Sci. 2002;19(7):727-34.

49. Evans DH, Piermarini PM, Choe KP. The multifunctional fish gill: dominant site of gas exchange, osmoregulation, acid-base regulation, and excretion of nitrogenous waste. Physiol Rev. 2005:85(1):97-177.

50. Henderson IW, Hazon N, Hughes K. Hormones, ionic regulation and kidney function in fishes. Symp Soc Exp Biol. 1985;39:245-65.

51. Whittamore JM. Osmoregulation and epithelial water transport: lessons from the intestine of marine teleost fish. J Comp Physiol B, Biochem, Syst, Environ Physiol. 2012;182(1):1-39
52. Tse WK, Au DW, Wong CK. Characterization of ion channel and transporter mRNA expressions in isolated gill chloride and pavement cells of seawater acclimating eels. Biochem Biophys Res Commun. 2006;346(4):1181-90.

53. Tse WK, Au DW, Wong CK. Effect of osmotic shrinkage and hormones on the expression of $\mathrm{Na}+/ \mathrm{H}+$ exchanger-1, $\mathrm{Na}+/ \mathrm{K}+/ 2 \mathrm{Cl}$ - cotransporter and $\mathrm{Na}+/ \mathrm{K}+-$ ATPase in gill pavement cells of freshwater adapted Japanese eel, Anguilla japonica. J Exp Biol. 2007;210(Pt 12):2113-20.

54. Li Z, Lui EY, Wilson JM, Ip YK, Lin Q, Lam TJ, et al. Expression of key ion transporters in the gill and esophageal-gastrointestinal tract of euryhaline Mozambique tilapia Oreochromis mossambicus acclimated to fresh water, seawater and hypersaline water. PLoS ONE. 2014;9(1):e87591.

55. Gregorio SF, Carvalho ES, Encarnacao S, Wilson JM, Power DM, Canario AV, et al. Adaptation to different salinities exposes functional specialization in the intestine of the sea bream (Sparus aurata L.). J Exp Biol. 2013;216(Pt 3):470-9.

56. Huber RD, Gao B, Sidler Pfandler MA, Zhang-Fu W, Leuthold S, Hagenbuch B, et al. Characterization of two splice variants of human organic anion transporting polypeptide 3A1 isolated from human brain. Am J Physiol Cell Physiol. 2007;292(2):C795-806.

57. Peter MC. The role of thyroid hormones in stress response of fish. Gen Comp Endocrinol. 2011;172(2):198-210.

58. McCormick SD, Bradshaw D. Hormonal control of salt and water balance in vertebrates. Gen Comp Endocrinol. 2006;147(1):3-8.

59. Rivera C, Voipio J, Thomas-Crusells J, Li H, Emri Z, Sipila S, et al. Mechanism of activity-dependent downregulation of the neuron-specific K-Cl cotransporter KCC2. J Neurosci. 2004;24(19):4683-91.

60. Kang KJ, Shibukawa Y, Szerencsei RT, Schnetkamp PP. Substitution of a single residue, Asp575, renders the NCKX2 K+-dependent Na+/Ca2+ exchanger independent of K+. J Biol Chem. 2005;280(8):6834-9.

61. Oudit GY, Kassiri Z, Sah R, Ramirez RJ, Zobel C, Backx PH. The molecular physiology of the cardiac transient outward potassium current (I(to)) in normal and diseased myocardium. J Mol Cell Cardiol. 2001;33(5):851-72.

62. Cerrone M, Delmar M. Desmosomes and the sodium channel complex: Implications for arrhythmogenic cardiomyopathy and Brugada syndrome. Trends Cardiovasc Med. 2014;24(5):184-90.

63. Cerrone M, Lin X, Zhang M, Agullo-Pascual E, Pfenniger A, Chkourko Gusky H, et al. Missense mutations in plakophilin-2 cause sodium current deficit and associate with a brugada syndrome phenotype. Circulation. 2014;129(10):1092-103.

\section{Submit your next manuscript to BioMed Central and take full advantage of:}

- Convenient online submission

- Thorough peer review

- No space constraints or color figure charges

- Immediate publication on acceptance

- Inclusion in PubMed, CAS, Scopus and Google Scholar

- Research which is freely available for redistribution

Submit your manuscript at www.biomedcentral.com/submit
C Biomed Central 